\title{
Dehnungsplastik nach spindelförmiger Exzision kombiniert mit einer modifizierten Insellappenplastik - die Mini-Insellappenplastik
}

\author{
Elliptical Excision Combined with Modified Island Pedicle Flap - The Mini Island Flap
}

Autor

Institut

\section{G. A. Barth}

Hautarztpraxis Schatz, Barth \& Degitz, München-Pasing

\section{Bibliografie}

DOI 10.1055/s-2007-966993

Online-Publikation:

21. 11.2007

Akt Dermatol 2007; 33 :

478-480 @ Georg Thieme

Verlag KG Stuttgart · New York ISSN 0340-2541

Korrespondenzadresse

Dr. med. Georg A. Barth

Hautarztpraxis Schatz,

Barth \& Degitz

Pasinger Bahnhofsplatz 1

81241 München

barth@hautpraxis.de

\section{Zusammenfassung}

$\nabla$

Die Dehnungsplastik nach spindelförmigen Exzisionen ist eine häufig eingesetzte Methode zur Defektdeckung in der operativen Dermatologie/ Dermatochirurgie. Nicht selten ist die Spannung an den Wundrändern, trotz Dehnungsplastik so hoch, daß man diese nicht durchführen kann ohne spannungsbedingte Komplikationen zu ris-

\section{Einleitung}

$\nabla$

Dehnungsplastiken sind eine häufig eingesetzte Methode zur Defektdeckung in der operativen Dermatologie/Dermatochirurgie [1-3]. In der Hand des Geübten sind dies einfache, schnelle, gewebeschonende und vielfältig einsetzbare rekonstrukive Verfahren. Ferner führen sie in der Regel zu ästhetisch ansprechenden Ergebnissen [4].

Trotz ausreichender Lappenbildung (Dehnungsplastik), kommt es in bestimmten Fällen zu einer sehr hohen Wundrandspannung im zentralen Defektbereich. Dies kann zu spannungsbedingten Wundheilungsstörungen führen. Ferner kann es zu spannungsbedingten Verziehungen von anatomisch wichtigen Strukturen (wie Lidern, Nasenflügeln etc.) kommen.

Um aufwendige plastische Verfahren, teils mit auffälligen geometrischen Narbenlinien (klassische Insellappenplastik), oder spannungsbedingten Komplikationen zu vermeiden, haben wir die Dehnungslappenplastik mit einer kleinen spindelförmigen Insellappenplastik kombiniert (৫ Abb. 1).

In unserem Fall führte diese Technik zu einem guten ästhetischen und komplikationslosen Ergebnis. kieren. Wir berichten über ein kombiniertes Vorgehen aus Dehnungslappenplastik und kleiner Insellappenplastik (Mini-Insellappenplastik), um die starke Spannung an den Wundrändern in der Mitte des Hautverschlusses zu reduzieren. Die modifizierte Operationstechnik führt in unserem Fall zur komplikationsloser Wundheilung und zu einem guten ästhetischen Resultat.

\section{Kasuistik und Methode}

$\nabla$

Bei der 71-jährigen Patientin wurde ein Basalzellkarzinom an der linken Schläfe kreisförmig exzidiert ( $\bullet$ Abb. 2). Die weiteren Inzisionslinien stellen Burow-Dreiecke dar, wie bei einer spindelförmigen Exzision. Zwei Mini-Insellappen wurden hier im dorso-kaudalen und fronto-kaudalen Burow-Dreieck umschnitten und subkutan gestielt. Nach weiterer subkutaner Lappenbildung nach allen Seiten (Dehnungsplastik) wurde der Defekt durch korial versenkte Nähte (resorbierbar, monofil, Polydioxanon) so verschlossen, dass der spannungsreiche Zentralbereich noch unverschlossen bleibt. Zentral wird der gestielte spindelförmige Mini-Insellappen kutan spannungsfrei eingenäht. In diesem Fall wurde der dorso-kaudale Mini-Insellappen wegen der geringeren Spannung am Lappenstiel verwendet, der zweite Lappen wurde verworfen. Die Hautfäden wurden am 7. postoperativen Tag gezogen. Der postoperative Heilungsverlauf war über 8 Monate komplikationslos ( Abb. 3).

\section{Diskussion \\ $\nabla$}

In der Operativen Dermatologie/Dermatochirurgie bestehen ein Vielzahl von Möglichkeiten einen Defekt zu verschließen (Hautlappenplastiken, Hauttransplantate, Sekundärheilung). An 

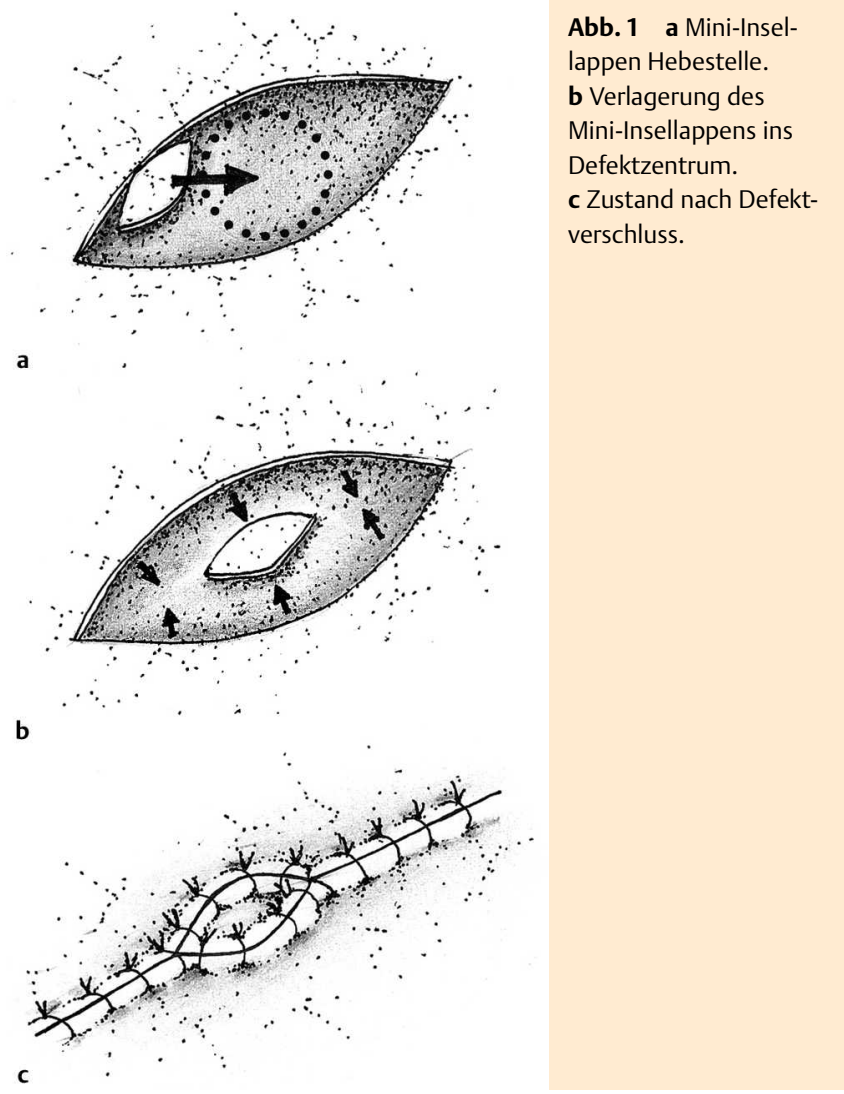

erster Stelle stehen die Aspekte der Funktionserhaltung, an zweiter die ästhetischen.

Wir stellen einen Fall vor, bei dem eine kombinierte Defektdeckung zweier Nahlappenplastiken zur Anwendung kam.

Durch die Kombination sind die Vorteile beider Hautlappenplastiken vereint worden.

Die Dehnungsplastik ist eine sichere, schnelle und ästhetisch ansprechende Hautlappenplastik. Allerdings besteht eine Limitierung bei starker zentraler Spannung [5]. Bei starker Wundrandspannung erhöht sich das Risiko für Wundrandnekrosen, Wundinfektionen, Dehiszenz, hypertrophe Narben/Keloide und Verziehungen mit funktionellen Beeinträchtigungen.

Der subkutan gestielte Insellappen ist ein bewährtes Hautoperationsverfahren [1,6,7], das das Problem der Wundrandspannung lösen kann. Lediglich die auffälligen, stark geometrischen (dreieckigen) Narbenlinien sind aus ästhetischer Sicht nicht immer zufriedenstellend [8]. Dies wird durch die hier vorgestellte MiniInsellappenplastik verhindert, weil der spindelförmige, kleine Lappen sich unauffällig in den Narbenverlauf entlang der Hautspannungslinien einfügt.

Durch die Modifikation, in Form des spindelförmigen Mini-Insellappens im Verlauf der Hautspannungslinien, wird ein gutes funktionelles und ästhetisches Ergebnis erzielt ( $\bullet$ Abb. 3).

Bei Defekten mit sehr großer Spannung und an kritischen anatomischen Stellen wird man auf die klassischen plastischen Vefahren aus der operativen Dermatologie/Dermatochirurgie zurückgreifen.

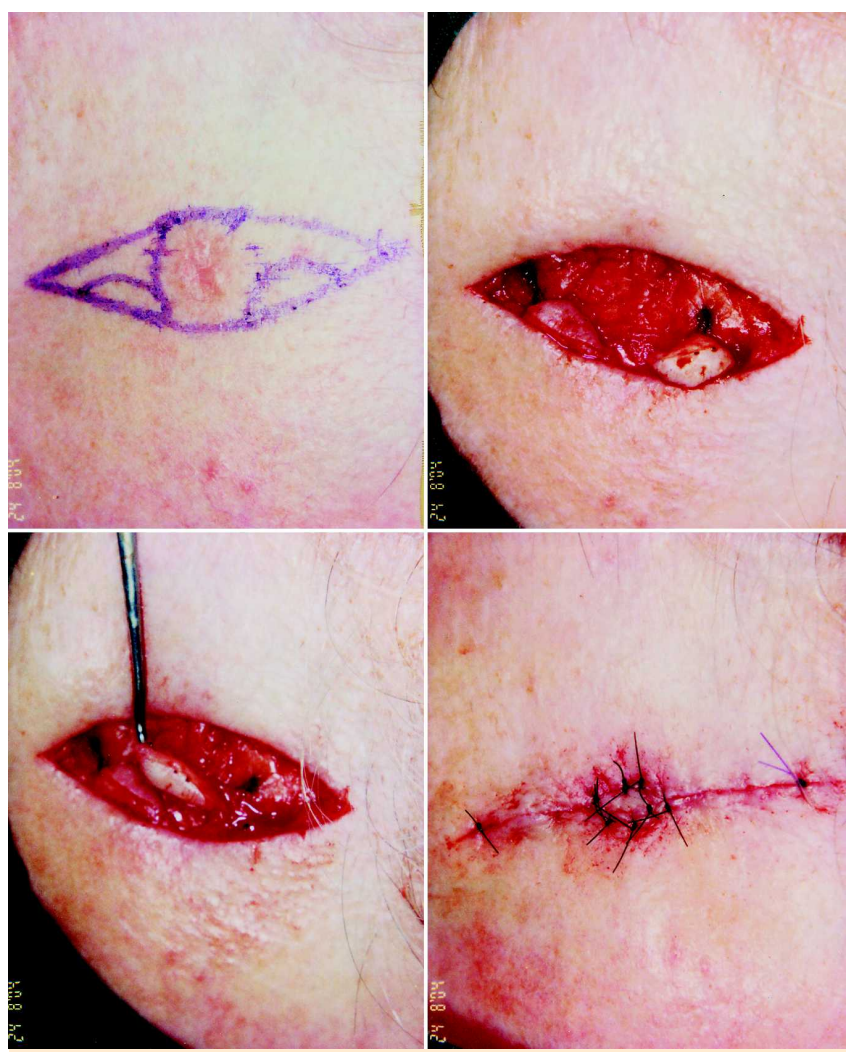

Abb. 2 Links oben: Einzeichnung der Operationsplanung. Rechts oben: Defekt nach Präparation und 2 Mini-Insellappen. Links unten: Verlagerung des gestielten Mini-Insellappens ins Zentrum des Defektes. Rechts unten: Postoperativ nach Defektverschluss.

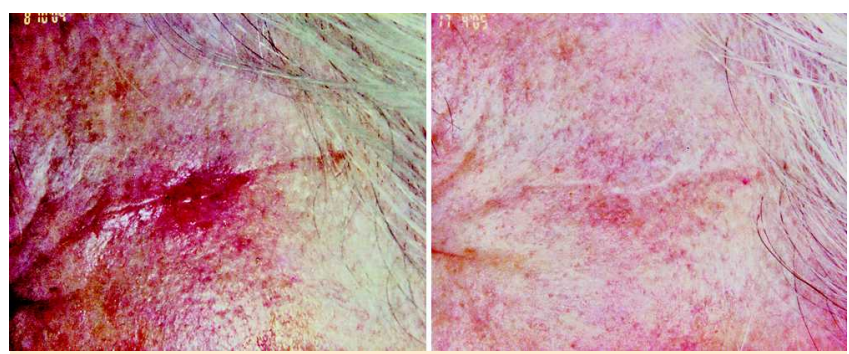

Abb. 3 Links: 6 Wochen postoperativ. Rechts: 8 Monate postoperativ.

\section{Abstract}

\section{Elliptical Excision Combined with Modified Island Pedicle Flap - The Mini Island Flap $\nabla$}

The elliptical excision is a widely used procedure in cutaneous surgery. Sometimes there is too much perpendicular tension in the central area to close the defect completely by a primary suture without risking complications by tension. We report on a combined elliptical excison and a modified island pedicle flap (mini island flap) to reduce high central perpendicular tension. Application of that modified operation technique leads to good wound healing and aesthetic result. 
Literatur

1 Petres J, Rompel R. Operative Dermatologie. 2. Auflage. Berlin: Springer, 2007

2 Kaufmann R, Podda M, Landes E. Dermatologische Operationen. 3. Auflage. Stuttgart: Thieme, 2005

3 Goldberg LH, Alam M. Elliptical excisions: Variations and the eccentric parallelogram. Arch Dermatol 2004; 140: 176- 180

4 Pardasani AG, Leshin B, Hallman JR, White WL. Fusiform incisional biopsy for pigmented skin lesions. Dermatol Surg 2000; 26: 622 -624

5 Boyer JD, Zitelli JA, Brodlang DG. Undermining in cutaneous surgery. Dermatol Surg 2001; 27: 75-78

6 Leonhardt JM, Lawrence N. Back to basics: The subcutaneous island pedicle flap. Dermatol Surg 2004; 30: 1787-1590

7 Hairston BR, Nguyen TH. Innovations in the island pedicle flap for cutaneous facial reconstruction. Dermatol Surg 2003; 29: 378 - 85

8 Skaria AM. Refinement of the island pedicle flap: Parallel placed release incisions to increase translation movement. Dermatol Surg 2004; 30: $1595-1598$

\section{Preisverleihung}

\section{Preis der Sächsischen Dermatologischen}

Gesellschaft e. V. 2007

Anlässlich der Jahresveranstaltung des Berufsverbandes der Deutschen Dermatologen e.V., Landesverband Sachsen am 17.11. 2007 in Leipzig, wurde zum 4. Mal der Preis der Sächsischen Dermatologischen Gesellschaft e.V. vergeben.

Mit diesem Preis werden besondere Leistungen auf wissenschaftlichem Gebiet, in Aus-, Weiter- und Fortbildung, in der Patientenbetreuung und außergewöhnliches Engagement in der Öffentlichkeitsarbeit sowie der Berufspolitik zur Förderung der sächsischen Dermatologen gewürdigt.

Der Preisträger war dieses Jahr Herr Dr. med. Michael Bär, Arzt in Weiterbildung an der Klinik und Poliklinik für Dermatologie an der Medizinischen Fakultät Carl-Gustav-Carus Dresden.

Herr Dr. Bär, ein junger Nachwuchs-Dermatologe, erhielt den Preis für sein Engagement in der dermatologischen Forschung und seine Publikation „Wavelength-Dependent Induction of CYP24A1-mRNA after UVB-Triggered Calcitriol Synthesis in Cultured Human Keratinocytes“, veröffentlicht im Journal of Investigation Dermatology 2007. 